\title{
A Comparison of Model-based Reasoning and Learning approaches to Power Transmission Fault Diagnosis ${ }^{1}$
}

\author{
Ramesh K. Rayudu \\ Dept. Natural Resources Eng.
}

\author{
Sandhya Samarasinghe \\ Dept. Natural Resources Eng.
}

\author{
Lincoln University, \\ Canterbury, \\ New Zealand. \\ rayudur@lincoln.ac.nz \\ sandhya@lincoln.ac.nz \\ kulasird@lincoln.ac.nz
}

\author{
Don Kulasiri \\ Ctr. for Computing \\ \& Biometrics
}

\begin{abstract}
An application of model-based reasoning and modelbased learning to an operative diagnostic domain such as electrical power transmission networks is presented. Most of the research in model-based diagnosis is based on maintenance diagnosis. Operative diagnosis, on the other hand, is done while the system is still in operation even after the fault. We plan to develop an efficient algorithm for operative diagnosis which can handle large domain of faults and multiple faults in real time. In our search toward a better algorithm, we develop and compare two different reasoning methods: diagnosis based on model based reasoning, and diagnosis based on heuristic rules learnt from model based reasoning. This paper presents the results of the comparison.
\end{abstract}

\section{Introduction}

Operative diagnosis (OD) of a physical system is the process of detecting faults while it is in operation whereareas in maintenance diagnosis (MD) [1], the fault diagnosis is done offline. Operative diagnosis is needed for systems which cannot be stopped for maintenance (as it is too expensive), and the diagnosis involves the consideration of symptoms and state which can change with time. In electrical power transmission networks, operative diagnosis is confined to alarm readings in real time while the effects of the faults are still propagating through the network. OD is heuristic in nature and often provides a challenging task for experts involved. Experts find that the pattern recognition of alarms triggered by a fault in the system is relatively easier task compared to the identification of the physical origins of the fault from a list of alarms. This difficulty could be due to several components malfunctioning at the same time within the network.

Power transmission networks carry power from supply utilities to the consumer and any fault in the network directly affects the consumers. The hazards of performing fault diagnosis in this domain incorrectly and too slowly result in notable accidents such as 1977 New York City blackout where the power restoration took several minutes causing inconvenience to consumers. Earlier research [2] has shown that decision-support systems can aid system controllers during emergency situations. This paper presents the proceedings of our attempt to develop an efficient fault diagnostician for power transmission network in New Zealand. Towards this development, model based techniques are applied to the domain and the results are presented in this paper.

\section{Model Based Diagnosis}

Model based diagnosis (MBD) is a form of diagnostic reasoning which incorporates operational principles of the devices in the form of models where the problem can be simulated under ideal conditions and the output (predicted) is compared with the observed output [1]. This comparison would reveal useful information for problem solving such as the status of the equipment in the power system network

\footnotetext{
${ }^{1}$ The funding from Trans Power NZ Ltd., New Zealand, for the project is greatly appreciated.
} 
and the authenticity of information reaching the control centres. Figure 1 shows operation of the fault diagnosis system using the model based approach.

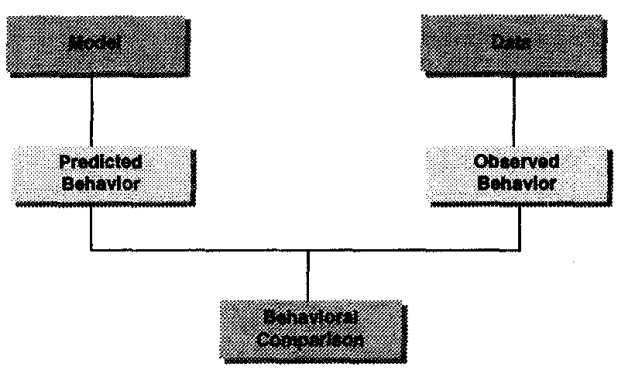

Figure 1. Model based reasoning.

As model based diagnosis or MBD is reasoning based on system model's behavioural comparison, it does not use any heuristic information about system failures [1]. Due to this reason model based diagnosis can be more advantageous over heuristic reasoning. Some of these advantages are listed below [10]:

$>$ MBD covers a wide range of fault scenarios than heuristic reasoning because $\mathrm{MBD}$ is based on the system's behavioural analysis.

$>\mathrm{MBD}$ can detect deviations from the expected behaviour.

$>\mathrm{MBD}$ can detect malfunctioning equipment in the early stages.

$>\mathrm{MBD}$ can predict the effects of the faults and unnecessary alarms as it simulates the faults.

$>$ MBD can handle multiple faults efficiently because the cascading effects of the faults can be simulated and analysed.

$>$ Adopt rule-based system's goal driven reasoning [10].

\section{Fault Diagnosis In Power Transmission Networks}

A prototype model based system for fault analysis and diagnosis (MoBFAD) of electrical power transmission was developed and was implemented in Prolog on LINUX/486. The motivation for its development was to produce and test a generic diagnostic system for electrical power networks. MoBFAD has a generic reasoning mechanism and can be setup to work for a particular network by encoding the model of that power network in the system.

MoBFAD is based on Reiter [3] and de Kleer, et al.'s [4] definition of model based diagnosis represented as a 3-tuple (system description, components and observations) and Igor Mozetic's [1] hierarchical model based diagnosis. The advantage of Reiter's approach is that it can infer diagnoses in terms of components from a set of observations. The important feature of these diagnoses is that they are minimal $^{2}$. Hierarchical structure is employed because the power system can easily be divided into different levels. In addition, hierarchical model based diagnosis is efficient to handle complex systems with large number of components or states of components and this helps a great deal when multiple faults are considered for diagnosis.

New Zealand's power transmission network comprises 13,005 route $\mathrm{Kms}$ of both $\mathrm{AC}$ and $\mathrm{DC}$ power cables and transmits around $30,000 \mathrm{Gw}$ of power per annum. For a network of this size, time and accuracy plays a very important role on its fault diagnosis. Solving the diagnostic problem in hierarchical levels has proven [5] to be faster than conventional model based reasoning. Diagnosis in MoBFAD is done in 3 levels:

1. Observation level.

2. Synopsis level.

3. Abstract level.

Observation level is the level where the components are observed for their behaviour. Any change in the network is reflected on this level; therefore, it is always active. The components are checked almost immediately for their operation and the component operation information is then passed onto synopsis level (level 2) where it is used to derive more abstract diagnoses. Abstract level (level 3) is a generalised heuristic level where the domain experts' knowledge is encoded and is used in the diagnosis at "network" level. Abstract level also has heuristic knowledge relating to problems (for example, cable faults) which do not need detailed diagnosis. Figure 2 shows the organisation of MoBFAD's hierarchical levels.

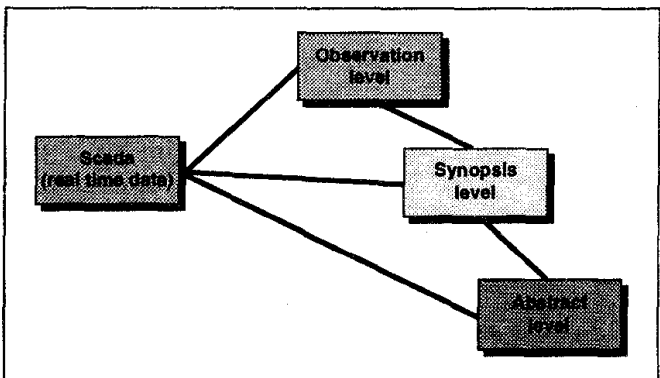

Figure 2. Hierarchical modelling in MoBFAD.

\footnotetext{
2 A minimal diagnosis is such that by changing a status of any abnormal component to normal would make the diagnoses inconsistent with the observations.
} 


\section{Goal driven reasoning in MoBFAD}

MoBFAD starts with "abstract" level by trying to solve the problem with its shallow reasoning knowledge. Observation and Synopsis levels are triggered by changes in the network and hence they operate in real time. If the shallow reasoning process of "abstract" level cannot find a solution, then the outputs (components' behaviour) of "observation" and "synopsis" levels are considered for the diagnosis. The behavioural component of the model is expressed as rules describing its qualitative properties. The model does not deal with quantitative representation of the components; instead, it symbolically describes the components' characteristic features. Examples of qualitative modelling can be found in [5] and [6] where the technique is used to model the electrical activity of heart and electrical power components, respectively.

An example of alarms input to MoBFAD would illustrate how model based reasoning works in practice. When the alarm list is input to MoBFAD, the list is analysed by "observation" level to find any malfunctioning of the components. Then heuristic rules are applied from the "abstract" level to solve the problem using shallow reasoning. If the problem could not be solved using shallow reasoning, then rules in "synopsis" level use the output from "observation" level to deduce some preliminary diagnosis. This preliminary diagnostic output is then used by "abstract" level to arrive at a solution.

Depending upon the problem, MoBFAD takes 0.13 to 2.5 secs (with multiple fault alarms) to arrive at a diagnosis. It is inferred that problems which can be solved using shallow reasoning are solved faster than model based reasoning. This brings our focus on using machine learning algorithms to learn. from simulated output of models and create generalised heuristic rules. The algorithm would search through the logical aspects of the reasoning process and record the common search paths by generalising them. The work done by several others has also prompted our utilisation of machine learning techniques. Mozetic $[5]^{4}$ discusses the compression of rules using Induction and Fattah \& O' Rorke[7] ${ }^{5}$ discuss the learning of association rules from models using Explanation based learning. Due to our domain's generalised nature, we chose to investigate some algorithms based on explanation based learning [8].

\footnotetext{
${ }^{3}$ Tests were conducted with same alarm list on both reasoning techniques. Model based reasoning took 0.24 secs to find the faulty device where as shallow reasoning took 0.19 secs to solve the same problem.

4 This research is applied to operative diagnosis.

${ }^{5}$ This research is applied to maintenance diagnosis.
}

\section{Model Based Learning}

Model based learning can be done in two ways:

1. learning knowledge by analysing the goal driven reasoning of model based reasoning [7].

2. learning meta-knowledge from existing knowledge using a qualitative model of the domain [8].

The first method was used to develop the learning mechanism. Two machine learning algorithms which are generally based on Explanation Based Generalisation (EBG), Peter Clark's "Lazy Partial Evaluation" (LPE) [8] and Mitchell et al.'s "Explanation Based Generalisation" (EBG) [9], were chosen for this purpose.

\section{Lazy Partial Evaluation}

Lazy Partial Evaluation (LPE) is a learning algorithm which is a hybrid of explanation based generalisation (EBG) and partial evaluation (PE) algorithms. Learning of LPE is same as that of EBG but, it also includes PE's ability to generalise and store the failed proofs. LPE replaces the original theory by a more generalised equivalent thereby making the runtime of the solution faster. The main advantages of LPE over EBG are [8]:

$>$ LPE eliminates EBG's repeated computation as it saves the total work done in exploring proofs other than the main proof.

$>$ LPE's "alternative proof saving" eliminates EBG's "masking effect" "which enhances the quality of the solution.

\section{Explanation Based Generalisation}

Explanation based generalisation is an articulation of the common aspects of various explanation based learning systems [9]. It is based on generalisation of a proof for a positive example by synthesising an operational definition of the proof in terms of its subgoals. The new definitions thus created are then added to the domain for future reasoning.

\section{Model based learning in MoBFAD}

Learning in MoBFAD is primarily based on Fattah and O'Rorkes' [7] proposal of integrating EBG and model based reasoning. However, their model is based

\footnotetext{
6 The Masking Effect of EBG is its inability to find alternative solutions as this may sacrifice the learning of most efficient solution; EBG learns only one solution it finds at the first instance.
} 
on maintenance diagnosis and need several additions. Our system adds the "state of the system" and learns the association rules with respect to MoBFAD's model hierarchy. In general, the learned rules will be of the following format:

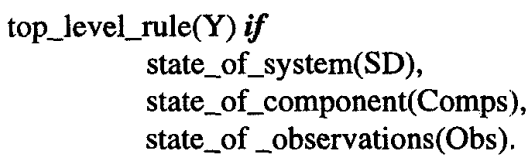

The variable $\mathrm{Y}$ is a function of logical variables appearing in the rule.

The resulting systems of this integration of machine learning algorithms and model based reasoning are called MoBFAD ebg $_{\text {(EBG }+M B R) \text { and }}$ MoBFAD $_{\text {lpe }}(\mathrm{LPE}+\mathrm{MBR})$. Both algorithms learn rules in advance.

\section{Comparative Results And Analysis}

To evaluate the algorithms, seven training (4 positive, 3 negative) examples are chosen. The results are shown in Table 1 . MoBFAD lpe generates fewer hypotheses and goal expansions than MoBFAD $\mathrm{D}_{\text {ebg. }}$.

Table 1. Comparative performance of algorithms ${ }^{7}$.

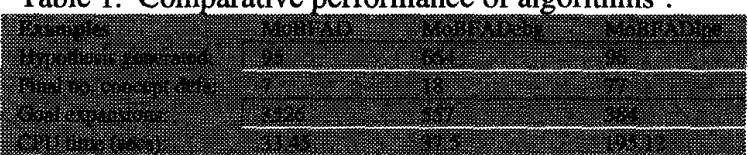

\section{CPU time}

Figure 3 shows the graph of CPU time utilised by each algorithm to solve ${ }^{8}$ all seven examples. All the examples for this analysis are taken from only one range of problems (a range with no pilot wire faults and observations are incomplete). During this analysis, the state of the power system is also considered constant. Under these circumstances, MoBFAD $\mathrm{D}_{\mathrm{pe}}$ takes more time to solve the proofs, but for the rest of the examples it used less time to solve. This is because MoBFAD $D_{\text {lpe }}$ reformulates the original definitions into several definitions using some initial examples and uses them on the following examples. On the other hand, MoBFAD ${ }_{\text {ebg }}$ does not use much time to learn in the initial stages, but it incrementally learns with the

7 The performance comparison is based on Peter Clark's criteria [8].

${ }^{8}$ Solving in here relates to algorithms' attempt to satisfy the goal and learn concept definitions (rules) simultaneously. number of examples. Eventhough MoBFAD $_{\text {lpe }}$ is slower in the initial stages, it is more efficient than the other algorithms. MoBFAD $\mathrm{Dbg}_{\text {eg }}$ is also efficient than MoBFAD but sometimes it uses more time than MoBFAD as in example 5 (Figure 3).

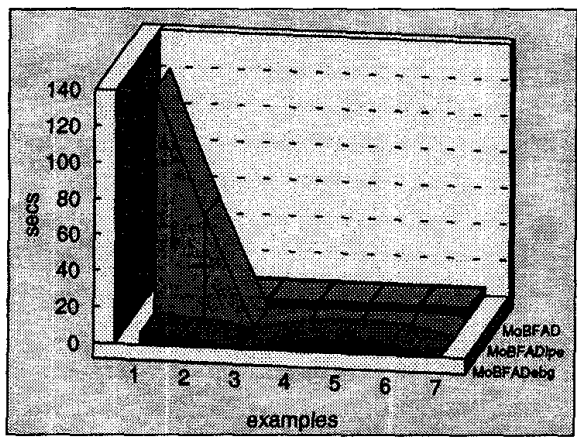

Figure 3. Performance graph of CPU-time.

\section{Number of rules learnt}

From the CPU time graph, it can be estimated that MoBFAD lpe learns more rules than MoBFAD ebg. For a given example, MoBFAD ${ }_{\text {lpe }}$ learns more concept definitions (see figure 4) than MoBFAD ${ }_{\text {ebg. This is }}$

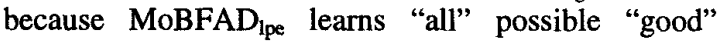
explanations of the example and it also learns some "not relevant" explanations along with explanations which lead to "failures". MoBFAD $D_{\text {ebg }}$ learns only the explanations which satisfy the example. Hence its upgradation of rules is linear. MoBFAD $\mathrm{D}_{\text {lpe, on the }}$ other hand, replaces the original definitions with new ones and this changes number of rules accordingly.

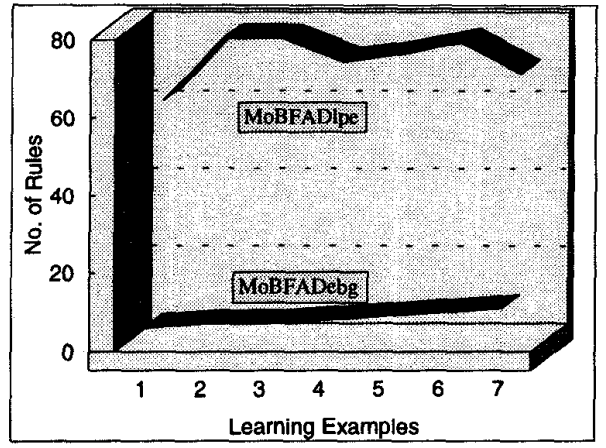

Figure 4. Graph of number of rules learnt.

\section{Discussion}

The above tests are done by considering constant "state" of the power system network. The need for this assumption is to test the efficiency of the algorithms 
under conditions used in other papers. Figure 5 shows the performance of each algorithm under dynamic conditions of power system state. The examples were chosen from a wider range of problems. According to the graph, reasoning with model based learning algorithms is faster than model based reasoning. On the other hand, it is also evident that the number of inferences used to find a solution increases with the increase in the number of rules. $M o B F A D_{\text {lpe }}$ is a preferred algorithm for fault diagnosis under constant network state but due to its longer learning time, it is not recommended for networks under dynamic conditions. In addition, $\mathrm{MoBFAD}_{\mathrm{lpe}}$ may not be able to deal with larger domains such as power transmission networks efficiently [8]. MoBFAD ${ }_{\text {ebg }}$ is consistent under both (constant and dynamic) conditions of the network but clearly demonstrates its inability to learn an efficient solution to the problem (see example $3 \& 5$ in Figure 5). At this stage, it can be concluded that MoBFAD ebg is preferred over MoBFAD lpe $_{\text {end }}$ MoBFAD because $\mathrm{MoBFAD}_{\text {ebg }}$ takes less time on average to arrive at a solution.

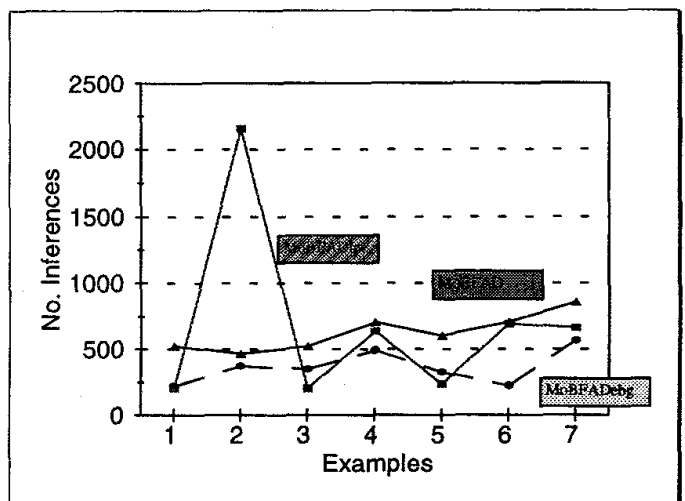

Figure 5. Performance graph of all algorithms under operative diagnostic conditions.

\section{Conclusion}

We have presented three algorithms applied to operative diagnosis based on model based reasoning and machine learning. MoBFAD, a pure model based system, is based on Igor Mozetic's Hierarchical Model Based Diagnosis and incorporates the domain models in three levels. MoBFAD lpe and MoBFAD ${ }_{\text {ebg }}$ are hybrid systems combining model based reasoning and machine learning. The machine learning algorithms, LPE and EBG, are used to learn top level rules from the model based diagnosis. All three algorithms are compared and their performance analysed. The hybrid algorithms work faster than model based diagnosis unless the top level rules grow unacceptably large. Under dynamic system conditions however, $\mathrm{MoBFAD}_{\text {ebg }}$ seems to provide the required solution faster.

\section{Acknowledgments}

Thanks to Peter Clark for letting us use his Prolog version of $L P E$ and $E B G$ algorithms.

Thanks to Control Centre Staff at SICC \& NICC, Trans Power NZ Ltd. for their feedback and support.

Thanks to Dr. Eduardo Morales of Institute of Electric Power, Mexico and Dr. Rambabu Adapa of EPRI, USA for their help and support.

\section{References}

1. Mozetic I., Hierarchical Model-Based Diagnosis, Intl. Jnl. Man-Machine Studies, 35 (3), 1991, 329-362. 2. Wollenberg B. F., Feasibility study for an Energy Management System Intelligent Alarm Processor, IEEE Trans. On Power Systems, May 1986, pp 241247.

3. Reiter R., A Theory of Diagnosis from First Principles, Artificial Intelligence 32, 1987, pp 57-95.

4. Johan de Kleer \& Williams B. C., Diagnosing Multiple Faults, Artificial Intelligence 32, 1987, pp 97130.

5. Mozetic I., Diagnostic efficiency of deep and surface knowledge in KARDIO, AI in Medicine 2 (2), 1990, pp 67-83.

6. Pfau-Wagenbauer M., and Nejdl W., Integrating Model-based and heuristic features in a real-time Expert System, IEEE-EXPERT, August 1993, pp 1218.

7. El Fattah Y. \& O'Rorke P., Explanation-Based Learning for Diagnosis, Machine Learning, 13,1993, pp 35-70.

8. Clark P. \& Holte R., Lazy Partial Evaluation: An integration of explanation based generalisation and partial evaluation, Proc. 6th European Conf. On Machine Learning (ECML-93), 1993, pp 360-365.

9. Mitchell T. M., Keller R. M., and Kedar-Cabelli S. T., Explanation based generalisation: A unifying view, Machine Learning, 1, 1987, pp 47-80.

10. Wang X., Dillon T., "A second generation expert system for fault diagnosis", Electrical Power \& Energy Systems, Vol 14, No 2/3, April/June 1992, pp 212-216. 